MUZIKOLOŠKI ZBORNIK - MUSICOLOGICAL ANNUAL XL

UDK 78:323.1

Ivan Florjanc

Akademie für Musik, Universität in Ljubljana

Pontificio Istituto di Musica Sacra, Roma

Akademija za glasbo Univerze v Ljubljani

Papeški inštitut za duhovno glasbo, Rim

\title{
Übernationales und nationales in der Musik
}

\section{Nadnacionalno in nacionalno $\mathrm{v}$ glasbi}

\author{
"Regnum unius linguae uniusque moris \\ fragile et imbecille est." \\ Ein Königreich, das aus einer Sprache und einer Kultur besteht, \\ ist zerbrechlich und schwach. \\ Heiliger Stefan, Ungarischer König
}

Beim Erforschen von nationalen und übernationalen Segmenten in der Musik ist der interdisziplinäre Zugang verpflichtend. Alle drei Begriffe in der Überschrift bleiben - trotz eines anderen Anscheins - immer noch ohne deutlicheren Definitionen. Der Begriff Nation hat sich lediglich in den letzten Jahrhunderten schon mehrmals verändert. Heute aber sind wir Zeugen von mehrschichtigen Identifikationsprozessen. C. L.-Strauss hat den Begriff Rasse ausgehöhlt und ihn mit Kultur gleichgesetzt, in der die Sprache die Rolle der ausschlaggebenden Diskriminante spielt. Die linguistische Analogie ist daher ein greifbares methodologisches Modell auch bei der Erforschung der Musik auf einem bestimmten geographischen Gebiet. Die nationalen und übernationalen Segmente (auch Segmente der Musik) auf einem geschlossenen geographischen Gebiet - wie z. B. Mitteleuropa - werden so langsam mit Hilfe einer Art kulturkundlicher Psychoanalyse in das Bewusstsein eindringen, d. h. mit Hilfe des Prozesses, bei dem durch das Vergleichen von unzähligen Angaben jenes ins Bewusstsein gerufen wird, was in den einzelnen Kulturen unund unter-bewusst bzw. selbstverständlich ist. Diese Arbeit ist - außer einigen wenigen Ausnahmen - noch nicht getan.
Pri študiju nacionalnih in nadnacionalnih segmentov $v$ glasbi je meddisciplinski pristop obvezen. Vsi trije pojmi v naslovu ostajajo kljub drugačnemu videzu še vedno brez določnejših definicij. Pojem narod se je v zadnjih stoletjih že večkrat spreminjal, danes pa smo priča večplastnim identifikacijam. C. L.-Strauss (1971) je pojem rase, ki je bil pri definiranju nacije v 20. stoletju odločilen, identificiral s kulturo. Znotraj nje pa je po njegovem odločilna diskriminanta zlasti jezik. Lingvistična analogija je zato priročen metodološki model tudi pri študiju glasbe na zaokroženem geografskem področju. Nacionalni in nadnacionalni segmenti (tudi glasbeni) na področju kot npr. Srednja Evropa bodo lahko prikapljali $\mathrm{v}$ zavest $s$ pomočjo neke vrste kulturološke psihoanalize, to pomeni s procesom, ko ob primerjavi številnih podatkov prinašamo $\mathrm{v}$ zavest tisto, kar je v posameznih kulturah ne- in pod-zavestno, oziroma samoumevno. Razen par izjem še to delo ni storjeno. Srednja Evropa, kjer se na malem prostoru stikajo številni narodi - torej kulture, je tudi kot enota odigrala $v$ novejši zgodovini v več ozirih ključno vlogo. Zato lahko pomeni edinstven ne le glasbeni ampak širok kulturološki primer, ki mu je vredno posvetiti študijsko pozornost tudi $z$ vidika sedanjih in prihodnjih družbenih odločitev. 
MUZIKOLOŠKI ZBORNIK • MUSICOLOGICAL ANNUAL XL

Auf dem Symposium, das die musikalische Identität Mitteleuropas zum Thema hat, ist es notwendig drei Schlüsselbegriffe, auf denen unsere Erörterungen beruhen, zumindest in globalen Umrissen zu streifen: übernational, national und Musik. Normalerweise sind wir überzeugt, dass jeder dieser Begriffe klar und eindeutig ist und wir daher darunter ein und dieselbe Sache verstehen bzw. wir stellen uns vor, dass wir ein und dasselbe Begriffsverständnis davon haben. Es ist aber sofort ersichtlich, dass dem nicht so ist. Alle drei Begriffe stammen aus einem Bereich, der mit ganz persönlichen, tiefgründigen und obendrein äußerst intimen Schichten verflochten ist, die zwangsläufig den Begriffsinhalt bestimmen. Ein anschauliches Beispiel dafür ist das Wort „Mama«: Dem Anschein nach handelt es sich im ersten Augenblick um einen objektiven Begriff, dennoch lässt sich aber schnell erkennen, dass jeder in den Wortinhalt die eigenen Erfahrungen, die eigene Bedeutung hineininterpretiert. Abstraktionen sind zwar auch beim Begriff „Mama" möglich und notwendig, jedoch stellt sich hierbei die Frage, womit wir es dann zu tun haben? Die Begriffe übernationales, nationales und Musik verhalten sich sehr ähnlich.

Es ist nicht notwendig, die komplexe und komplizierte Gesellschaftsstruktur Mitteleuropas gesondert hervorzuheben. Man kann sie bereits mit der folgenden schlichten Tatsache anschaulich darstellen, die auch ein Teil meiner persönlichen geschichtlichen Erfahrung ist. Meine Mutter ist heute 94 Jahre alt. In all ihren Jahren lebte sie bereits in fünf verschiedenen Staaten, obwohl ihr ständiger Wohnsitz immer derselbe geblieben ist: Österreich-Ungarn, altes Jugoslawien, Deutschland (Drittes Reich), neues Jugoslawien und heute Slowenien. Dasselbe erlebten in dieser Zeit die Bewohner von mindestens zehn verschiedenen Nationen Mitteleuropas. Sie leben auf verhältnismäßig kleinem Gebiet, der von Wien - dem einstigen und heutigen idealen Zentrum Mitteleuropas - kaum ein Paar hundert bis maximal tausend Kilometer entfernt ist. Die Plejade der Nationen und der Nationalitäten ist im Herzen Mitteleuropas nun einmal eine ersichtliche und unmittelbar wahrnehmbare Gegebenheit, die von niemandem negiert werden kann.

Die Suche nach nationalen und übernationalen Elementen in der Musik ist daher auf diesem kleinen zugleich aber äußerst vielfältigen Raum Mitteleuropas alles andere als eine leichte und einfache Arbeit. Jede Behauptung über die Präsenz von dem oder jenem nationalen Element in der Musik, beziehungsweise jede Behauptung über den über-nationalen Charakter der Musik eines bestimmten Komponisten bzw. von der oder jener geschichtlichen Stilrichtung beruht daher zwangsläufig auf normalerweise nur stillem, unbewusstem manchmal aber sogar verschwiegenem subjektivem Verständnis, das wir in die Wörter wie Nation, national/übernational, nationale Kultur, typisch "unsere" Musik oder typisch "unsere" Kultur u. ä. hineininterpretieren. Dieser Beitrag hat die Absicht auf einige grundlegende Fragen aus diesem Bereich aufmerksam zu machen. In jeder Wissenschaft ist die richtige Fragestellung von vorrangiger Bedeutung, um zu Antworten zu gelangen, die zeitlich und wertmäßig das Sekundärstadium des Forschungsprozesses darstellen.

Wenn wir das Wort Nation unter die Lupe nehmen, das den Wortstamm des eigenschaftswörtlich gebrauchten Wortpaares national/übernational bildet, stellen wir 
sofort fest, dass es eine Menge von Begriffen gibt, die das Wort „Nation“ in den letzten ungefähr drei- bis vierhundert Jahren enthalten haben. Es geht um den Zeitabschnitt, als sich das geografische und nationale Bild Mitteleuropas etabliert und die Gestalt angenommen hat, die es in etwa noch heute hat.

In der Zeit des Rudolf II. und noch weit über seine Zeit hinaus war auf dem Gebiet des Mitteleuropas der Nationsbegriff aus der Renaissance bzw. dem Humanismus vorherrschend (1): Mit dem Wort Nation bezeichnete man damals die regionale, lokale oder soziale Zugehörigkeit, aber auch verschiedene Körperschaften. Von nationaler Zugehörigkeit in dieser Zeit zu sprechen, ist daher noch ohne Bedeutung. (2) Erst das 18. Jahrhundert (Vico, von Muralt, Voltaire, Herder) legte die Grundlage für ein Verständnis, in das sich der Begriff, der sich zur Zeit der französischen Revolution herausgebildet ha, wie ein Edelreis einpflanzte: Die Nation verstanden als kollektive Einheit bzw. als Volk, mit dem politischen Selbst-Bewusstsein, ein legitimer Träger der Staatshoheit zu sein - im Gegensatz zum Monarchen oder den privilegierten Schichten. (3) Die Zeit, die wir üblicherweise als das Zeitalter der Romantik bezeichnen, ist voll mit intellektuellen Anstrengungen (Fichte, M. de Stael, Manzoni, Mazzini, Prešeren u. a.), die Charakterzüge dessen, was wir als Nation benennen, abermals mehr oder weniger erfolgreich zu definieren. In dieser Zeit tritt die Sprache als maßgebliches Kriterium in den Vordergrund. Die Idee von der Sprache als grundlegende Diskriminante beim Definieren der Nation verbindet sich in der damaligen Zeit eng mit der politischen Kultur des liberalen, demokratischen aber auch konservativen Charakters. Eine Mischung aus diesen Zügen war der Nährboden für die ideologische Aufladung zahlreicher Nationalbewegungen im Europa des 19. Jahrhunderts. Insbesondere im deutschsprachigen Umfeld haben sich in dieser Zeit zwei Bedeutungen für den Begriff Nation gebildet: Kulturnation und Staatsnation. Das Gewimmel neuer Staaten am Ende des ersten Weltkrieges, die sich nach dem nationalen Prinzip formiert haben, wurde von der mehrmals kontradiktorischen Weiterentwicklung der Idee Nation im Einklang mit den ideologischen und kulturellen Hauptströmungen der Zeit einschließlich jener begleitet, die von internationalem Charakter waren wie z. B. der Liberalismus, der Sozialismus, der Kommunismus. Ein schöner zeitlich bedingter Begriffsabglanz dieser Entwicklung ist die Bezeichnung der damals - im Jahre 1919 - neu gegründeten internationalen Gemeinschaft der Nationen, der am 1. Januar 1942 die Neugründung der Vereinten Nationen (ONU) folgte, die noch heute unter der gleichen Bezeichnung existiert und alle Völker der Erde umfasst.

Die ideelle und praktische Zusammenfügung der Begriffe Nation und Staat in der zweiten Hälfte des 19. Jahrhunderts und am Anfang des 20. Jahrhunderts, ließ die Nationalismen aufkeimen und sich ausbreiten, die wir uns bis heute auch in Mitteleuropa mit ansehen. Viele machen darauf aufmerksam (F. Chabod), dass im Phänomen der Nationalismen logischerweise und geschichtlich gesehen Ideenmodelle gegenwärtig sind, die naturalistische Komponenten wie z. B. das Land, die Rasse, die Sprache u. ä. beinhalten. Widerstandsfähiger gegen die Gefahr des Nationalismus sollten aber jene Nationen sein, die voluntaristische Züge in den Vordergrund stellen, die sich in Form von freien Wahlen äußern. Zahlreiche Ereignisse in der Geschichte beweisen, dass so eine Unterscheidung gegenstandslos ist: Oft schon waren 
MUZIKOLOŠKI ZBORNIK - MUSICOLOGICAL ANNUAL XL

wir Zeugen, dass jedes noch so hohe Ideal bzw. Idee in der Lage war, in gegebenen geopolitischen und geschichtlichen Situationen ein starkes nationalistisches Potential bei zahlreichen Völkern in Europa und der Welt in Gang zu setzen.

Auf theoretischem Gebiet hat man, was den Nationsbegriff betrifft, versucht zwischen der Nation als éthnos und der Nation als demos zu unterscheiden. Mit ethnos soll die kulturelle, linguistische und andere ähnliche Zugehörigkeit gemeint sein. Mit dem Begriff Nation, verstanden als demos, soll hingegen insbesondere die Staatsangehörigkeit, einschließlich aller Rechte, Pflichten und der Loyalität gegenüber der demokratischen Verfassung gemeint sein. Es geht um eine Unterscheidung, die sich auf das bereits vorhin erwähnte Begriffsverständnis von Nation als Kulturnation und Staatsnation stützt. Beide Auffassungen gehören vor allem in den deutschsprachigen Raum.

Die heutige Situation überall in Europa, die wir als das Phänomen des Sich- $Z u$ sammen-Schließens auf höherem über/staatlichen Niveau und der stark zunehmenden Immigration von Menschen verschiedenster Rassen und aus allen Kontinenten miterleben, stellt neue Herausforderungen und führt zu neuen Erscheinungen. Es wird immer deutlicher, dass Nationalstaaten - geschichtlich gesehen - lediglich eine eigenartige Klammer sind, obwohl die "Religion" der Nationalstaaten ihre eigenen Märtyrer hat, genauso wie der Glaube. Auf jeden Fall aber ist es immer offensichtlicher, dass Nationalstaaten von kürzerer Dauer sind als die Religion, die einen Teil der Menschheit ausmacht. Domovina/očetnjava/Vaterland/patria usw. sind Begriffe, wofür vor noch nicht all zu langer Zeit - auch mit Hilfe emotionaler Unterstützung der Mütter - Tausende ihr Leben gegeben haben. Heute aber lösen gerade dieselben Begriffe bei einzelnen Personen eines bestimmten Staates den Prozess einer neuen Identitätssuche, einer Identitätserneuerung, einer radikalen Vervollständigung der persönlichen Ausweiskarte aus.

Das altehrwürdige vereinte Mitteleuropa kann in so mancher Hinsicht ein so manches Lösungsmodell bieten. Wir müssen aber einige Jahrhunderte weit zurückgreifen und die damaligen Modelle gründlich und neu überdenken.

Heute ist es erforderlich den Begriff der nationalen Identität auf dem Gebiet des gesamten Europas zu vertiefen. Ähnlich wie seinerzeit auf dem Gebiet des Mitteleuropas ist es unabdingbar von übemationaler und untemationaler Identität im Bezug auf die nationale Identität zu sprechen. Die unternationale Identität tritt immer dort und dann in Erscheinung, wo bzw. wenn das übernationale Identität aufkeimt bzw. im Begriff ist sich zu bilden. Auch hier ist es wiederum notwendig, das Verhältnis eines Teiles im Bezug auf das Ganze auf allen Ebenen zu definieren: auf der Ebene der Staaten, der Völker, religiöser und Rassengruppierungen u. ä. Es geht um das Definieren des Verhältnisses zwischen partikulär und allgemein und umgekehrt. Im Römischen Reich war es der Pantheon, der die beiden Dimensionen partikulär-allgemein, Teil-Ganzes sanktioniert und mit Erfolg bewertet hat. Jeder Gott eines noch so kleinen Volkes hatte im Pantheon in Rom seinen genau bestimmten Platz, was damals mit Hilfe von religiösen Symbolen die Anerkenntnis heutiger Begriffe von nationaler und persönlicher Identität bedeutete. 
Der erwähnte Prozess der neuerlichen und mehrschichtigen Identifikation (C. Ginzburg) ist heute ein vielfältiges und äußerst stark verästeltes Geschehen. Ein sehr festes Kriterium dabei können verschiedene Perspektiven des Zugehörigkeitsgefühls sein:

- Identifikation auf Basis der Assimilation: So mancher Slowene war z. B. ein stark assimilierter Jugoslawe, jedoch der Angriff auf Slowenien im Jahre 1992 hat dieses spezifische Zugehörigkeitsgefühl vollkommen zunichte gemacht. Oder: Die Österreicher haben z. B. mit dem Anschluss einen Teil ihrer spezifischen Zugehörigkeit verloren, wie auch die Slowenen, Kroaten, Tschechen, Juden u. a. sie verloren haben, doch die Rassengesetze, die bei den anderen den Verlust der spezifischen Zugehörigkeit bewirkt haben, haben die Österreicher nicht berührt. Das Zugehörigkeitsgefühl der Österreicher wurde dadurch nicht beeinträchtigt. Meine Mutter fühlte sich als Österreich-ungarische Slowenin und nicht als slowenische Staatsangehörige der Österreich-ungarischen Monarchie und erinnert sich auch heute noch gern an den Österreich-ungarischen Staat ungeachtet dessen, dass ihr Bruder und ihr Vater im ersten Weltkrieg schwer verletzt wurden. Das Dritte Reich ist ihr völlig fremd geblieben.

- Identifikation auf Basis der Rassenzugehörigkeit: Die Identifikation aufgrund des Blutes, des Erdbodens, des Landes u. ä. kommt insbesondere dort vor, wo Menschen in ein und demselben Ort geboren werden und auch sterben, $d$. h. in der Gesellschaft derselben Menschen. Das Gleichsein und die Zugehörigkeit werden in so einem Umfeld als etwas Naturgegebenes erlebt. Heute geht dieses Zugehörigkeitsgefühl infolge der starken Migration zusehends zurück.

- Identifikation auf Basis der sprachlichen Zugehörigkeit: Dieser Identitätstypus ist vor allem an die Muttersprache gebunden, an jene Sprache, in der jemand denkt/ spricht, zählt/rechnet usw.

- Identifikation auf Basis der geographischen Zugehörigkeit: Mitteleuropa hatte und hat hier immer noch einige günstige Möglichkeiten.

- Identifikation auf Basis eines pluralistischen Zugehörigkeitsgefühls: Das multiple Zugehörigkeitsgefühl ist heute wieder eine sehr häufige und verbreitete Erscheinung.

- Schamgefühl als Kriterium der Identifikation: Die Schamhaftigkeit, das Schamgefühl als starkes Zugehörigkeitskriterium - das geschieht immer dann, wenn ich mich schäme etwas zu tun, weil und sofern ich beispielsweise Slowenien - früher Jugoslawien - angehöre (aus meinem Blickwinkel betrachtet). In diesem Sinne ist folgende Frage bedeutsam: Wer schämt sich heute, wenn ein Europäer etwas Böses anrichtet? Ebenso kann ich mich schämen, wenn jemand aus meinem Kreis, dem ich angehöre, etwas Schlechtes macht. Die Scham kann ein sehr starker, wenn nicht der stärkste Indikator des Zugehörigkeitsgefühls sein. Der Zorn hingegen kann als Indikator der Nichtzugehörigkeit herangezogen werden.

Der, der den Prozess der neuerlichen Identifikation bzw. der Um-Identifizierung verwirklicht, ist schließlich und endlich eine Einzelperson, ein Individuum, ein Subjekt, das daher als Knotenpunkt des Geflechts bzw. als Schnittpunkt unterschiedlichster Mengen, einzelner Gesamtheiten und Texturen begriffen werden kann: eine Art Geistes- und Gewissensparlament, dem das sogenannte Ich den Vorsitz führt. 
MUZIKOLOŠKI ZBORNIK • MUSICOLOGICAL ANNUAL XL

Beispiel: Ich stamme aus dem Savinja-Tal, bin ein Slowene, war Staatsbürger von Jugoslawien und Italien, bin männlich und Teil der Menschheit, habe einen Bart und bin von heller Hautfarbe, spreche die slowenische Muttersprache, bin katholischer Christ, Demokrat und Liberaler sowie Pazifist (wenn ich aber physisch angegriffen werde, kann ich mich im $\mathrm{Nu}$ in einen Texaner mit einer Pistole verwandeln), bin Komponist, Professor für die Kompositionslehre, Erforscher des Menschen und seiner geistigen Fähigkeiten insbesondere auf dem Gebiet der Musik u. ä., dennoch kann ich auf keines dieser Merkmale reduziert werden. Am wenigsten aber kann meine Identität auf den Fingerabdruck, die Regenbogenhaut oder den genetischen Abdruck beschränkt werden. Und das ungeachtet der Tatsache, dass mich gerade das am genauesten physisch bestimmt bzw. am tiefsten in meinen Körper reicht, in mein physisches Ich.

Intellektuelle Anstrengungen im letzten halben Jahrhundert haben einige Denkstützpunkte aufgestellt, die versucht haben auf rationalem Wege die Rückkehr der Irrwege beider Weltkriege zu verhindern. Claude Levi-Strauss hat im Rahmen dieser Anstrengungen Behauptungen herauskristallisiert, die heute ein allgemein anerkanntes Denkmodell darstellen: Würden wir innerhalb größerer Gruppierungen von Menschen Rassenunterschiede zu Beginn der Menschheitsgeschichte herausfinden wollen, würden wir uns (C. L.-Strauss, 1971) infolge derselben Tatsache in die Lage versetzen, dass wir nichts erfahren würden. Eine solche Untersuchung würde nicht die Rassenvielfalt, sondern die kulturelle Vielfalt aufdecken. Bei der Erforschung der Rassenunterschiede können wir uns auf den Grund des Körpers oder Geistes begeben, auf komplizierte Formen menschlichen Verhaltens, menschlicher Temperamente oder Rassen einlassen und werden immer nur auf kulturelle Unterschiede stoßen.

Diese Behauptung widerspricht nicht der Tatsache, dass unterschiedliche Kulturen auf gleichem Raum zusammenleben. Die Kultur einer Gruppierung ist diejenige, die vorgibt bzw. die Festlegungen von geographischen Grenzen, von freundschaftlichen oder feindlichen Beziehungen auch auf dem Gebiet des genetischen Austauschs mit Hilfe von Vermählungen hinnimmt. Die Rasse - folglich auch die einzelne Nation - ist schließlich und endlich nur eine von vielen Funktionen der Kultur. Die Kultur bestimmt die Rasse und nicht umgekehrt, denn sogar jede Kultur selektioniert biologische Merkmale, die wiederum auf die Kultur Einfluss nehmen. Bedeutsam ist in diesem Zusammenhang die Feststellung, dass die Genetiker alte Begriffe wie z. B. Rasse typ schon vor geraumer Zeit mit den Begriffen wie Volk oder Bevölkerung, und das Wort Rasse sogar mit der Bezeichnung genetischer Stock ausgetauscht haben.

Für unsere Diskussion, wenn wir diesen Gedankenweg fortsetzen, ist jener Gedanke sehr verführerisch, der sich direkt aus Lévy-Strauss' Feststellung ableitet: Es geht um die Möglichkeit des Vergleichens kultureller Modelle mit linguistischen, $\mathrm{d}$. h. mit gesprochener Sprache ${ }^{1}$. Es geht um die sogenannte linguistische Analogie.

1 It. linguaggio, engl. language, franz. langage, dt. Sprache. 
MUZIKOLOŠKI ZBORNIK • MUSICOLOGICAL ANNUAL XL

Lévy-Strauss hat die linguistische Analogie wortwörtlich genommen. In der gesprochenen Sprache ist es nicht möglich, die Bedeutung direkt aus einzelnen isoliert auftretenden Wörtern, herauszufinden und auch nicht indirekt aus der Lautanalyse dieser Wörter, obwohl im Aussagesatz beides vorkommt. Ähnlich kann die Bedeutung einer kulturellen Erscheinung erst durch das Vergleichen unzähliger Angaben, mit Hilfe des interkulturellen Vergleichs, wie das in der Psychoanalyse gemacht wird, herausgefunden werden: Durch das Vergleichen unzähliger Angaben kann jenes bewusst gemacht werden, was in den einzelnen Kulturen selbstverständlich, infolge im wortwörtlichen Sinne un- bzw. unter-bewusst ist. Lévy-Strauss setzt es mit l'esprit humain gleich, was aber anscheinend bereits schon ein inhaltlicher Doppelgänger zu Durkheims kollektiver Vernunft sein kann. Allem Anschein nach klopft Lévy-Strauss - im Unterschied zum Sozialwissenschaftler Durkheim - mit seinem l'esprit humain vor allem an die Tür der Ästhetik, es versteht sich - der Ästhetik im weitesten Sinne.

Es ist bekannt, dass die gesprochene Sprache zuerst aus Lauten besteht, die so zusammengesetzt sind, dass sie sich intim mit der menschlichen Atmung decken. Mit der Atmung decken sie sich sogar dann, wenn es lediglich um den Gedankengang ohne Worte geht. Die Sprachmodelle sind sowohl klanglich als auch begrifflich kodifiziert und können allseitig Betrachtungs- und Studiengegenstand sein. Gerade dieser Bereich ist durch umfangreiche Forscherbemühungen von Linguisten auf der einen Seite (sie sind insbesondere auf das klangliche, physische Erscheinungsbild der Sprache aufmerksam) bis hin zu Grammatikern auf der anderen Seite (sie sind insbesondere auf das begriffliche, die Physikalität überschreitende Erscheinungsbild aufmerksam) abgedeckt. Eine ähnliche Forschungsatmosphäre durchdringt die Anthropologie und die Musikwissenschaft: die einen schenken mehr Aufmerksamkeit den sichtbaren und "greifbareren" Erscheinungen, für die anderen ist die abstrakte bzw. mentale Hintergrundseite derselben Erscheinungen verlockender. ${ }^{2}$

Beinahe ein Jahrhundert währende strukturalistische Untersuchungen und Studien haben uns gemeinsam mit den Forschungsergebnissen aus der Phonologie die Funktionsweise phonematischer Modelle weitgehend klar gemacht ${ }^{3}$. Die Bezeichnung (lat. significatio) bzw. die Bedeutung ist demnach mit einer bestimmten Auswahl unter unzähligen möglichen Geräuschen und Klängen sowie deren gegenseitigen Kombinationen beauftragt, die durch die menschliche Stimme erzeugt und wahrgenommen werden können. Mit anderen Worten: Eine bestimmte Bedeutung ist eng verbunden - wenn nicht im bestimmten Maße sogar schon abhängig - von ihren intensitätsmäßigen, höhenmäßigen, zeitlichen, mengen- und qualitätsmäßigen Klangnuancen. Das gilt für alle Sprachen und nicht nur für jene aus dem fernen Osten, die im musikalisch-klanglichem Sinne äußerst feinfühlend sind.

\footnotetext{
2 Einst sagte man dazu einfach: physisch und metaphysisch.

${ }^{3}$ Insbesondere die gründliche Forschungsarbeit Jakobsons auf dem Gebiet der Phonetik und seine linguistischen Untersuchungen hatten einen starken Einfluss auch auf die Ethnomusikwissenschaft und die Musikwissenschaft.
} 
Die blutsverwandtschaftliche Verbindung ersten Grades mit der Musik ist von selbst ersichtlich und muss nicht gesondert bewiesen werden.

Hier entspringt vielleicht auch jenes Bedürfnis nach enger Verbindung im Zusammenhang des allumfassenden Wissens der Alten zwischen der Grammatik, der Rhetorik, der Dialektik, der Musik, aber auch der Arithmetik, der Geometrie und der Astronomie. ${ }^{4}$ Dieses Wissen nannte man Weisheit und jede dieser Wissenschaften war stolze und selbstbewusste Dienerin auf ihrem Hofstaat, was für die damaligen Begriffe bedeutet, dass jede von ihnen von adeliger Abstammung war. Die Musik war, wie wir gesehen haben, gleichwertiges - bei einigen Autoren sogar zentrales Mitglied der allumfassenden göttlichen Weisheit, sapientia divina, wobei erstaunlicherweise die Poesie ausgeschlossen war und das mit verächtlichen Beinamen infima doctrina, was auf deutsch in etwa folgendes bedeuten würde: minderwertige oder sogar niederträchtige Wissenschaft. ${ }^{5}$ Dieses Primat hat die Musik unter den Wissenschaften verloren, als sie immer mehr zum Malen mit Klängen verkommen ist, eine Art Klangmalerei geworden ist und keine Wissenschaft mehr war. Diese Selbst-Degradierung der Musiker in der modernen Geschichte hat aber Rolle der Musik innerhalb des menschlichen Betätigungsfeldes, das wir so gerne als Kultur bezeichnen, nicht geschmälert. Hier knüpfen wir an die eingangs erwähnten Gedanken an. Jedoch kehren wir lieber zu unserer Analogie zurück, weil sie uns noch einige Schritte tiefer führen kann.

Die Musik nimmt auch heute noch ihren Platz unter den Wissenschaften ein, obwohl ihre damalige Rolle in andere Räumlichkeiten übersiedelt ist. Ich denke vor allem an die Rolle der Phonetik in der Linguistik und darüber hinaus in der Semiologie bzw. Semiotik. Schon allein die Rede, das Gespräch, das Streiten und Scherzen, das Weinen und Lachen u. ä. ist eine Art musikalischer Akt, der in musikalischer Ver/ kleidung auftaucht und wir ihn mit dem Organ, das wir als Gehör ${ }^{6}$ bezeichnen, entkodifizieren.

Lediglich phonetisch betrachtet (was leicht auf das Biologische zurückgeführt werden kann), entsteht der Anschein, dass alle Sprachen einander sehr ähnlich sind, einige zumindest sogar fast gleich. Jedoch dieser einfachen Tatsache steht die konträre aber für alle evidente Tatsache gegenüber: nämlich der aus/gesprochene Gedanke besteht niemals nur in der nebeneinander in bestimmten an den Atemzug

${ }^{4}$ Diese Verbindung ist präsent seit den Griechen über das gesamte Mittelalter hinweg (im Grunde geht es um den weitreichend Einfluss der karolingischen Reform) bis hin zu den Humanisten und darüber hinaus bis zu den Aufklärern, in verändertem Sinne sogar bis heute.

5 Im Mittelalter stellte in diesem Zusammenhang für die damaligen Denker die Frage, warum sich die göttliche Wissenschaft in der Bibel poetischer Bilder bedient, eine sehr verzwickte Angelegenheit dar. Darüber spricht Thomas von Aquin in deutlicher und verdichteter Form in seinem Werk Summa Th., I, 1, 9.: "Utrum sacra scriptura debeat uti metaphoris".

${ }^{6}$ Insbesondere die Verwendung des Wortes Gehör ruft in unserem kollektiven Bewusstsein eine allgemeine aber typisch musikalische Erscheinung hervor, dessen wir uns normalerweise nicht bewusst sind: jemandem Gehör schenken, Gehör für Menschen haben, sich Gehör verschaffen, Gehör finden, zu Gehör bringen u. ä. beinahe fast gleich wie jemand hat ein absolutes musikalisches Gehör. 
gebundenen Abständen erfolgenden Aufstellung und Anordnung von Phonemen sowie morphologischen Elementen (Morphemen). ${ }^{7}$ Der aus/gesprochene Gedanke ist auf den ersten Blick wahrlich vor allem ein verästeltes grammatikalisches Gebilde, das die Bedeutung (lat. significatio, Bezeichnung) mit Hilfe des Aneinander-Reihens von Klängen und Geräuschen bzw. Wörtern und deren rhetorischen Intonationen $^{8}$ vermittelt. Unter den Linguisten gibt es bis heute berechtigterweise kein Einvernehmen über die Existenz von universellen Grundsätzen in der Grammatik, noch weniger über die Art und den Typus dieser Grundsätze.

Die Analysen der Linguisten haben sich bis vor kurzem fast ausschließlich nur auf sequentielle, d. h. lineare, syntagmatische bzw. metonymische Ordnung der wörtlichen Wiedergabe der Klänge konzentriert. In der Musik könnte das die Melodie bedeuten. Das ist vollkommen verständlich, denn gerade diese Gesichtspunkte sind in der rational gegliederten Rede vorherrschend, trotz eines anderen Anscheins. Die Linguisten wollten daher zuerst herausfinden, wie einige Abläufe von Wortgliederungen jene Stufe erreicht haben, dass es ihnen gelungen ist, einen Sinn zu erlangen. In dieser Hinsicht unterscheiden sich die Linguisten überhaupt nicht von den Anthropologen der Kultur der Boas-Schule. Die linguistische bzw. kulturelle Ganzheitlichkeit wäre demnach nur die einfache Summe des linearen Zählblattes sequentiell zusammengesetzter Teile, die wir einzeln wahrnehmen können. Die kulturelle bzw. sprachliche Aufsplitterung ist aber in Wirklichkeit eine sehr komplexe und komplizierte Erscheinung.

Parallel - beziehungsweise reziprok dazu - schenkten die Linguisten bisher weniger Aufmerksamkeit den synchronischen, symbolischen, paradigmatischen und metaphorischen Gesichtspunkten, die den Sinn direkt vermitteln können, d. h. vor allem mit der Synthese. Die Metapher bzw. das Sinnbild in der Rede kann mit der Harmonie in der Musik verglichen werden. Sie ist überwiegend vor allem in poetischen Mitteilungen sowie in magisch/religiöser Sprachverwendung präsent. ${ }^{9}$ Hier steckt vielleicht der Grund, warum es lediglich in der Musiksprache (sofern sie so bezeichnet werden kann) möglich ist, mehr Bedeutungen gleichzeitig auszudrücken (sofern auch diese so bezeichnet werden können). Aus diesem Grund ist die Musik scheinbar hermetischer und erweckt den Eindruck der Unnahbarkeit, wenn nicht

7 Das Verhältnis zwischen dem Phonem und der Bedeutung wird mittels der Kombinationsreihe des Wortes Roma schön deutlich gemacht, die in italienischer Sprache erst in der fünfzehnten (mora, Strafe bei verspäteter Zahlung) bzw. vierundzwanzigsten Kombination (amor, Liebe) Wörter erzeugt, die in italienischer Sprache einen Sinn ergeben:

Roma - ramo - rmao - roam - raom - rmoa

orma - oram - omar - omra - oamr - oarm

mroa - mrao - mora - moar - maor - maro

arom - armo - aorm - aomr - amro - amor

8 Mehr darüber in Chomsky, Syntactic Structures (1957) und in Language and Mind (1968). Dieses zweite Werk vgl. auch mit der lt. Übersetzung Mente e linguaggio, in: Chomsky, Filosofia del linguaggio, Torino, Boringhieri 1969, S. $131-245$.

9 In diesem Zusammenhang ist die geschichtliche Tatsache aus dem Bereich der Musik interessant, dass der musikalisch entwicklungsmelodische (lineare) Gesichtspunkt der Vorgänger des harmonischen (vertikalen) Aspekts ist, obwohl die Harmonie auch in der Zeit der Polyphonie und sogar der Monodie immer präsent war, wenn auch nur im Unterbewussten. Könnten wir in diesem Zusammenhang abschließend sagen, dass die Musik wieder einmal der zuvorkommende Moment der menschlichen Geistesentwicklung ist. 
manchmal sogar auch einer magischen Dimension. Jedoch darüber etwas mehr erst später. An dieser Stelle genügt schon die "geographische Angabe" darüber, wo sich jener Ort mit der Höhle befindet, die angeblich ins Jenseits führt - natürlich sinnbildlich ausgedrückt, d. h. "harmonisch", wenn wir so noch rasch bis zum Ende unsere Gedanken weiterspinnen. In unserem Fall bedeutet das Jenseits in erster Linie die "Welt der Musiksprache". Setzen wir daher diesen Weg fort, um zu sehen, wohin uns die linguistische Analogie noch führen kann.

Die Kultur kann somit, wie wir schon flüchtig erwähnt haben, vor allem als Kommunikation verstanden werden. Die Kultur kann nur über die sinnlich wahrnehmbare Textur des Einzelnen kommunizieren, der Empfänger/Sender kultureller Botschaften ist. Im Inneren der einzelnen Person, des Individuums geht es aber um Spannungen und Prozesse zwischen der Vernunft und dem Gehirn. Die Musik hat als bedeutsamer Teil der Kultur, sei es im Menschen selbst, sei es in Gruppen von Menschen, die ganz unterschiedlich bezeichnet werden können - auch mit nationalen Attributen - ebendiese Gesetzmäßigkeiten und ebendiese Funktionen.

Die Musik, obwohl wir bis heute ihre Definition nicht kennen, ist ausschließlich an den Klang und das Gehör gebunden, wenn es sich aber um Kunstmusik handelt auch an das Sehvermögen. In allen menschlichen Dimensionen versetzt uns die Musik heute auf dieselbe Art und Weise in Zeit und Raum, wie sich das den Pfahlbautenbewohnern des Sumpfgebiets um Ljubljana ereignet hat.

So wie die Sprache verschiedene Mundarten kennt, hat auch die Musik eigene Wege der Sinn- und Bedeutungsgebung. Wege, die - in erster Linie - an diese oder jene stilistische musikalische Form, an dieses oder jenes Klangmodell, manchmal sogar an diese oder jene Anordnung einzelner Stimmen gebunden sind. Diese Wege der Sinngebung sind eng an ein bestimmtes gesellschaftliches (soziologisches) und somit indirekt auch nationales Modell gebunden. Der Sinn, die Bedeutung, die Botschaft werden zwar empfangen, geformt, geboren - wie wir gesehen haben - aber auch angenommen und erfasst (percepire-recepire) zuerst in jenen menschlichen "Organen", wo der Gedanke, das Gewissen seinen Sitz haben bzw. wo die Freiheit und der Entscheidungsprozeß - das menschliche Ich also - wurzeln. Das gilt ebenso für das, was begrifflich ist - und daher mit Worten - eingefangen werden kann, als auch für das, was begrifflich ist - und daher mit Worten - nicht eingefangen werden kann. Zu dieser zweiten Kategorie zählt unter anderem fast zur Gänze auch die Musik. Obwohl es einerseits um nicht tastbare - quantitativ nicht messbare - Dinge geht, geht es aber andererseits um ein intimes Eingewebt-Sein in den Gemeinschaftskörper - und das regelmäßig, manchmal sogar auch in den gesamten Gesellschaftskörper. Nur über diesen Weg werden auch der Kern und der Inhalt der musikalischen Botschaft wahrnehmbar und fassbar, begrifflich und daher auch mit Worten einfangbar. Die Osmose in diesem Bereich kann am lebendigsten gerade in intimen und geschlossenen Gruppierungen von Menschen sein, z. B. in der Familie oder in primären Gruppen, wo die Membranen zwischen den Zellen dieser Grundgemeinschaft am dünnsten sind. 
MUZIKOLOŠKI ZBORNIK • MUSICOLOGICAL ANNUAL XL

Eine uralte mythologische Musiküberlieferung aus dem europäischen Osten vermittelt uns folgendes: Orpheus - ein wunderbarer Sänger - hat bei seiner Wiederbelebungsverrichtung nicht philosophiert, keinen Vortrag gehalten, nicht gegrübelt und getüftelt, nicht taktisch überlegt und auch nicht Gedichte vorgetragen. Er hat gesungen und gespielt. Er hat sich - seiner Meinung nach - des tauglichsten "Instruments" bedient, des Werkzeugs-Waffen, um auf seinem Weg die feindseligen Kräfte der Unterwelt zu bewältigen. Auch die Konzentration und die Wegrichtung ist es ihm gelungen, durch das Singen beizubehalten. Er durfte nämlich nicht nach rückwärts blicken. Interessant und bedeutsam ist auch der Wortstamm des Wortes Orpheus. Das griechische orfanós ist das lateinische orbo, was auf deutsch einsam, Einzelgänger bzw. auch der Arme bedeutet. In einigen Überlieferungen wird Orpheus sogar die körperliche Blindheit zugeschrieben, um so angeblich besser mit den Augen des Geistes sehen zu können.

Es ist kein bloßer Zufall, das die menschliche Stimme lange Zeit als die einzige Form der Musik verstanden wurde, bezeichnet als musica instrumentalis. In solch einer Anschauung steckt wahrlich die ideelle Auffassung aus der Vergangenheit, in der die Musik als eine Art Gerüst gesehen wurde, das Festigkeit verlieh und die Harmonie aufrecht erhielt in allem, was lebt ${ }^{10}$. Man sprach von der Harmonie der Himmelskörper, die angeblich sogar, für das Ohr nicht hörbare Musik, bezeichnet als musica mundana, erzeugt haben, was den Gleichklang des gesamten Universums, des Himmels und der Erde, bedeutete. Ferner sprach man von musica humana, was das menschliche Universum, seine kleine Welt - seinen Mikrokosmos - bedeutete. Die Musik in der Bedeutung, die sie heute hat, stand erst an der dritten Stelle: Man bezeichnete sie als musica instrumentalis. Damit war nicht die Instrumentalmusik gemeint, sondern die Musik, die durch die menschliche Stimmer erzeugt wird. Die menschliche Stimme ist nach dieser Auffassung das einzige Werkzeug (Instrument), das in der Lage ist der unhörbaren kosmischen und mikrokosmischen Musik Ausdruck zu verleihen. Obwohl uns heute die Auffassung in ihrer ursprünglichen Bedeutung nichts mehr sagt, kann sie für uns aber trotz allem in ihrer übertragenen Bedeutung sehr nützlich sein. Zum Glück hat die Allegorie erneut ihr eigenes Heimatrecht erlangt im Bezug auf das gesamte zeitgenössische menschliche Verhalten. Die Harmonie, die Ordnung im Menschen und in der Welt können zum gemeinsamen Nenner der Kunst und der Welt werden.

Ein altes Erkenntnisprinzip des Philosophen Aristoteles besagt: "Nihil in intellectu, quod non prius in sensu." Dasselbe in der Terminologie unserer Betrachtungsweise ausgedrückt, würde heißen: Die gesamte Musik ist ein Klang, den der Mensch zurechtgelegt hat im Einklang mit seinen eigenen inneren Geistesbewegungen nach seinem inneren Abbild, ähnlich wie der Schöpfer. Das gilt für jede Musik ohne Ausnahme. Eine gewisse Form dieser Klangordnung ist daher zwangsläufig an ein be-

\footnotetext{
${ }^{10}$ So dachte noch der Humanist J. Keppler (1571-1630), der in seiner astronomischen Abhandlung getrost den Begriff und Ausdruck harmonices mundi verwendet hat, wo es um die enge Verbindung harmonisch-mathematischer Gesetze als astronomische Grundlage des Kosmos gegangen ist. Auch der Philosoph Leibnitz spricht an der Schwelle zum neuen Zeitalter ohne Scham von der prästabilierten Harmonie.
} 
stimmtes Umfeld und an die Zeit gebunden. Sie widerspiegelt somit Modelle der einzelnen Person und der menschlichen Gemeinschaft, der eine bestimmte Musikart angehört und für die sie bestimmt sind. Sie kann uns daher viel sagen, sei es über die menschliche innere Textur selbst, sei es - andererseits - über die Textur seiner Kultur, der jeder Einzelne zugehörig ist.

Die Musik kann somit in der Tat - aus demselben Grund - einer der möglichen Wegen sein, um Erkenntnis darüber zu erlangen, wie eine bestimmte menschliche Gemeinschaft geordnet ist. Die Musik kann folglich die Rede der gesellschaftlichen Gruppierungen innerhalb der Menschheit sein. Eine Rede also, die Bezeichnungen (significatio), Bedeutungen, Botschaften beinhaltet. Dasselbe lässt sich aus dynamischem Blickwinkel ausdrücken: Die Musik ist die klangliche Abstimmung des Einzelnen und von Gruppierungen von Menschen untereinander, als Mittel des SichBewusst-Werdens kann sie eine außerordentliche Gelegenheit zur Beseitigung von Störungen in der allseitigen kulturellen Kommunikation, Disonation bzw. im allseitigen kulturellen Gleichklang werden. Denn die Musik offenbart - das können wir jetzt getrost versichern - das allertiefste Geheimnis über den Menschen und seine immensen Entwicklungsfähigkeiten, nämlich, dass die ursprünglichste Quelle jeder kulturellen Schaffenskraft jenes Bewusstsein ist, das aus der gesellschaftlichen Zusammenarbeit und dem Liebesaustausch erwächst.

Wenn die Behauptungen wahr sind, dass es keine Rassen gibt, sondern nur Kulturen, die gerade in den Sprachen ihre starke Diskriminante haben, wenn - außerdem - wahr ist, dass die Musik Sprache ist, dann wird es möglich sein, jene charakteristischen Merkmale herauszuschälen, die typisch sind für eine ganz bestimmte Kultur aber auch jene, die einer größeren Anzahl von kulturellen Einheiten gemeinsam sind. Die nationalen und übernationalen Segmente auf einem geschlossenen geographischen Gebiet - wie z. B. Mitteleuropa - werden so langsam mit geduldiger und beharrlicher kulturkundlicher Psychoanalyse in das Bewusstsein eindringen, $\mathrm{d}$. h. durch das Vergleichen von unzähligen Angaben wird jenes ins Bewusstsein gerufen, was in den einzelnen Kulturen un- und unter-bewusst bzw. selbstverständlich ist. Erst, wenn diese Arbeit gründlich gemacht werden wird zumindest von Einzelnen - Individuen - als Drehscheibe des mehrschichtigen Geschehens in der Gesellschaft, wird es möglich sein etwas Stichhaltigeres über nationale und übernationale (also gemeinsame) Identitäten mit Bezug auf die Musik zu sagen.

Auch auf diesem Gebiet hat folgender Merksatz seine Gültigkeit: In dem Augenblick, wo wir die Quelle der Musik in uns erleben werden, wird es uns nicht schwer fallen, aufzuhorchen und das Plätschern der Quelle in anderen zu respektieren. 